\title{
ON POSITIVE NORMALIZATION OF FRACTIONAL ORDER CONTINUOUS DISTURBANCE SINGULAR SYSTEM
}

\author{
Muhafzan \\ Department of Mathematics \\ Faculty of Mathematics and Natural Sciences \\ Universitas Andalas, Indonesia \\ muhafzan@sci.unand.ac.id

\section{Lyra Yulianti} \\ Department of Mathematics \\ Faculty of Mathematics and Natural Sciences \\ Universitas Andalas, Indonesia \\ lyra@fmipa.unand.ac.id
}

\section{Article history:}

Received 06.02.2019, Accepted 10.06.2019

\begin{abstract}
Recently, the fractional order continuous singular system becomes research field that much discussed by various researchers. The normalization constitutes a variant of discussing for fractional order singular system. The procedure to normalize positively of the fractional order continuous disturbance singular system is discussed in this paper. Some sufficient condition that guarantees the existence of a fractional derivative output feedback such that the closed loop system constitutes a fractional order usual linear system and positive, is established. The considered problem is solved by transforming it into a usual fractional order linear system, and afterwards it is analyzed using algebraic principle. The final result of this paper is a sufficient condition that guarantees the existence of a fractional derivative feedback such that the closed loop system constitutes a fractional order usual linear system and positive.
\end{abstract}

\section{Key words}

Positive normalization, fractional order, singular system, disturbance.

\author{
Admi Nazra \\ Department of Mathematics \\ Faculty of Mathematics and Natural Sciences \\ Universitas Andalas, Indonesia \\ admi@fmipa.unand.ac.id \\ Zulakmal \\ Department of Mathematics \\ Faculty of Mathematics and Natural Sciences \\ Universitas Andalas, Indonesia \\ zulakmal@fmipa.unand.ac.id
}

\section{Introduction}

Consider the following system:

$$
\begin{aligned}
\left(E \triangle^{\alpha}-A\right) \mathbf{x} & =B \mathbf{u}+C \omega \\
\mathbf{y} & =D \mathbf{x}
\end{aligned}
$$

where $\mathbf{x}$ is state vector, $\mathbf{u}$ is control vector, $\omega$ is disturbance vector, $\mathbf{y}$ is output vector, $E, A \in \mathbb{R}^{n \times n}, B \in$ $\mathbb{R}^{n \times m}, C \in \mathbb{R}^{n \times q}, D \in \mathbb{R}^{p \times n}$ and $\operatorname{rank}(E)=r<n$. In the system in (1), $\triangle^{\alpha}$ denotes the fractional-order derivative operator of order $\alpha$ in sense of Caputo with $0<\alpha<1$, which its definition is given in [Kilbas, Srivastava and Trujillo, 2006] as follows:

$$
\triangle^{\alpha} \mathbf{x}=\frac{1}{\Gamma(1-\alpha)} \int_{0}^{t} \frac{\dot{\mathbf{x}}(\tau)}{(t-\tau)^{\alpha}} d \tau
$$

where

$$
\Gamma(v)=\int_{0}^{\infty} e^{-t} t^{v-1} d t
$$

is the Euler Gamma function satisfying the functional equation $\Gamma(v+1)=v \Gamma(v)$. If $r=n$ and $\alpha=1$, the system 1 is the usual linear system. If $r=n$ and $0<\alpha<1$, the system (1) is a fractional order normal linear system [Chikriy, 2008]. The application of 
fractional order normal linear system is given in [Kaczorek, 2014] and [Metia, Oduro, Duc and Ha, 2016]. If $r<n$ and $\alpha=1$ then the system (1) is the usual singular (descriptor) system [Duan, 2010].

In this paper it is focused on the case $r<n$ and $0<\alpha<1$. The couple equation (1) is known as the fractional order disturbance singular system. Different to the fractional order normal linear system that always have a solution, the fractional order singular system may be not have a solution for some situation. It has a unique solution if there exists a complex number $\lambda$ such that $\operatorname{det}(\lambda E-A) \neq 0$ and in this case it is called regular [Kaczorek and Borawski, 2016].

As shown in [Kaczorek and Borawski, 2016], if $\operatorname{det}(\lambda E-A) \neq 0$ for some complex number $\lambda$ then there exists some nonsingular matrices $Q_{1}, P_{1} \in \mathbb{R}^{n \times n}$ such that

$$
Q_{1}(\lambda E-A) P_{1}=\lambda\left[\begin{array}{cc}
I_{r} & O \\
O & N
\end{array}\right]-\left[\begin{array}{cc}
J & O \\
O & I_{n-r}
\end{array}\right]
$$

where $J \in \mathbb{R}^{r \times r}$ is a Jordan matrix and $N \in$ $\mathbb{R}^{(n-r) \times(n-r)}$ is a nilpotent matrix. Let

$$
Q_{1} B=\left[\begin{array}{l}
B_{1} \\
B_{2}
\end{array}\right], Q_{1} C=\left[\begin{array}{l}
C_{1} \\
C_{2}
\end{array}\right], D P_{1}=\left[\begin{array}{ll}
D_{1} & D_{2}
\end{array}\right]
$$

where $B_{1}, B_{2}, C_{1}, C_{2}, D_{1}$ and $D_{2}$ are the matrices of suitable size. Under the transformation

$$
\mathbf{z}=P_{1}^{-1}\left[\begin{array}{l}
\mathbf{z}_{1} \\
\mathbf{z}_{2}
\end{array}\right]
$$

the system (1) can be written as

$$
\begin{aligned}
\left(\triangle^{\alpha}-J\right) \mathbf{z}_{1} & =B_{1} \mathbf{u}+C_{1} \omega \\
\left(N \triangle^{\alpha}-I_{n-r}\right) \mathbf{z}_{2} & =B_{2} \mathbf{u}+C_{2} \omega \\
\mathbf{y} & =D_{1} \mathbf{z}_{1}+D_{2} \mathbf{z}_{2}
\end{aligned}
$$

where $\mathbf{z}_{1} \in \mathbb{R}^{r}$ and $\mathbf{z}_{2} \in \mathbb{R}^{n-r}$.

Different to the fractional order usual linear system, the solution of fractional order singular system may contain impulse that appear from the solution of the second equation of (5). It is well known that the impulse constitutes an unwanted behavior because it may cause degradation in performance or even destroy the system [Duan, 2010]. In literature [Goncharova, Samsonyuk, Staritsyn, 2017] and [Matviychuk, 2018] it is stated that the impulse must be controlled, some even claim that impulse needs to be removed. Therefore it is important to eliminate this impulse behavior. For the usual singular system, the impulse can be eliminated by normalization process [Duan and $\mathrm{Wu}, 2005]$, that is, by using a state feedback of the form

$$
\mathbf{u}=-K_{1} \triangle \mathbf{x}
$$

for some matrix $K_{1} \in \mathbb{R}^{m \times n}$. Using this state feedback, the system (1) becomes

$$
\left(\left(E+B K_{1}\right) \triangle-A\right) \mathbf{x}=\mathbf{0} .
$$

It is clear that if $\operatorname{rank}\left(E+B K_{1}\right)=n$, then the system (6) is a usual linear system. Some condition for the existence of the matrix $K_{1}$ such that $\operatorname{rank}\left(E+B K_{1}\right)=$ $n$ has been reported in [Duan, 2010].

In this paper the concept of normalization for the usual singular system is extended to the fractional order disturbance singular system, by constructing a fractional derivative output feedback of the following form:

$$
\mathbf{u}=-\left(K_{E} \triangle^{\alpha}-K_{A}\right) \mathbf{y}
$$

where $K_{E}, K_{A} \in \mathbb{R}^{m \times p}$ are the gain matrices to be determined. Using (7) the system (1) becomes the following closed loop system:

$$
\left(\left(E+B K_{E} D\right) \triangle^{\alpha}-\left(A+B K_{A} D\right)\right) \mathbf{x}=B \omega .
$$

It is obvious that if $\operatorname{rank}\left(E+B K_{E} D\right)=n$, then the system (8) is a fractional order usual linear system and it is free impulse. It is a task in this paper to establish the criteria to ensure the existence of the matrix $K_{E}, K_{A} \in \mathbb{R}^{m \times p}$ such that $\operatorname{rank}\left(E+B K_{E} D\right)=n$.

In the application field in which the system (1) appears as a model of some real problems, the positiveness of the state variable is a must. A fractional order usual linear system is called positive if for all $t>0$, it holds $\mathbf{x} \in \mathbb{R}_{+}^{n}$ and $\mathbf{y} \in \mathbb{R}_{+}^{p}$ for each control $\mathbf{u} \in \mathbb{R}_{+}^{m}$ and $\omega \in \mathbb{R}_{+}^{p}$ [Kaczorek, 2008]. It is well known that if $r=n$ and $\alpha=1$, the system (1) is positive iff $E^{-1} A$ is a Metzler matrix, $E^{-1} B \in \mathbb{R}_{+}^{n \times m}, E^{-1} C \in \mathbb{R}_{+}^{n \times q}$ and $D \in \mathbb{R}_{+}^{p \times n}$ [Muhafzan and Stephane, 2013]. This criterion holds for the case $r=n$ and $\alpha<1$ as well [Kaczorek, 2008]. It is obvious that if $E^{-1} A \in \mathbb{R}_{+}^{n \times n}$ then $E^{-1} A$ is a Metzler matrix. Therefore, the objective of this paper is to establish a criterion that ensure the existence of matrices $K_{E}, K_{A} \in \mathbb{R}^{m \times p}$ such that $\operatorname{rank}\left(E+B K_{E} D\right)=n$ and the closed loop system (8) is positive. To the best of the authors knowledge, there is no research yet on how to normalize a fractional order singular continuous system.

This paper is organized as folows. Section 2 discusses some useful materials related to the desired results. The procedure to normalize the fractional order continuous disturbance singular system positively is discussed in section 3. A numerical example is presented to illustrate the result. We end this study by the conclusion which is given in section 4 .

\section{Some Useful Results}

In order to find the desired goal, some the following results is useful. 
Definition 1. [Kaczorek, 2008] A matrix $A \in \mathbb{R}^{n \times n}$ is said to be monotone if it is nonsingular and $A^{-1} \in$ $\mathbb{R}_{+}^{n \times n}$. A matrix $M \in \mathbb{R}_{+}^{n \times n}$ is said a monomial matrix if there exist exactly one nonzero entry in every row and column of $M$.

It has been proved in [Kaczorek, 2008] that the matrix $A^{-1} \in \mathbb{R}_{+}^{n \times n}$ if and only if $A$ is a monomial.

Definition 2. [Kaczorek, 2008]

1. Two matrices $M, S \in \mathbb{R}_{+}^{n \times n}$ is said positively equivalent if there exists two monomial matrices $P, Q$ such that $S=Q M P$.

2. A matrix $M \in \mathbb{R}_{+}^{n \times n}$ with rank $M=s$ is said $s$ monomial if it is positively equivalent to the matrix $\operatorname{diag}\left(M_{S}, O\right)$, where $M_{S}$ is monomial.

The $s$-monomial matrices have the following properties.

Theorem 1. [Kaczorek, 2008]

1. The matrix $M$ is s-monomial iff $M$ has $(n-s)$ rows and column with entries equal to zero and $s$ rows and column with only one entry different to zero.

2. If the matrix $M$ is s-monomial then it is positively equivalent to the matrix $\operatorname{diag}\left(I_{S}, O\right)$.

\section{Positive Normalization}

In this section we present the desired results. First of all, it will be generated $K_{E}, K_{A} \in \mathbb{R}^{m \times p}$ such that $\operatorname{rank}\left(E+B K_{E} D\right)=n$. Assume that the matrix $E$ is positively equivalent to $s$-monomial matrix $M$, then there exists some monomial matrices $Q$ and $P$ such that

$$
Q E P=\left[\begin{array}{cc}
M_{S} & O \\
O & O
\end{array}\right]
$$

Moreover, let

$$
Q A P=\left[\begin{array}{ll}
A_{1} & A_{2} \\
A_{3} & A_{4}
\end{array}\right], Q B=\left[\begin{array}{l}
B_{1} \\
B_{2}
\end{array}\right], D P=\left[\begin{array}{ll}
O & D_{2}
\end{array}\right]
$$

where $A_{1} \in \mathbb{R}^{s \times s}, A_{2} \in \mathbb{R}^{s \times(n-s)}, A_{3} \in$ $\mathbb{R}^{(n-s) \times s}, A_{4} \in \mathbb{R}^{(n-s) \times(n-s)}, B_{1} \in \mathbb{R}^{s \times m}, B_{2} \in$ $\mathbb{R}^{(n-s) \times m}$ and $D_{2} \in \mathbb{R}^{p \times(n-s)}$. Assume that $D_{2}$ and
$B_{2}$ have full rank. Choosing $K_{E}=B_{2}^{\top} D_{2}^{\top}$, it is found

$$
\begin{aligned}
E+B K_{E} D= & Q^{-1}\left[\begin{array}{cc}
M_{S} & O \\
O & O
\end{array}\right] P^{-1} \\
& +Q^{-1} Q B K_{E} D P P^{-1} \\
= & Q^{-1}\left(\left[\begin{array}{cc}
M_{S} & O \\
O & O
\end{array}\right]\right. \\
& \left.+\left[\begin{array}{c}
B_{1} \\
B_{2}
\end{array}\right] B_{2}^{\top} D_{2}^{\top}\left[\begin{array}{ll}
O & D_{2}
\end{array}\right]\right) P^{-1} \\
= & Q^{-1}\left(\left[\begin{array}{cc}
M_{S} & O \\
O & O
\end{array}\right]\right. \\
& \left.+\left[\begin{array}{cc}
O & B_{1} B_{2}^{\top} D_{2}^{\top} D_{2} \\
O & B_{2} B_{2}^{\top} D_{2}^{\top} D_{2}
\end{array}\right]\right) P^{-1} \\
= & Q^{-1}\left[\begin{array}{cc}
M_{S} & B_{1} B_{2}^{\top} D_{2}^{\top} D_{2} \\
O & B_{2} B_{2}^{\top} D_{2}^{\top} D_{2}
\end{array}\right] P^{-1} .
\end{aligned}
$$

Since $D_{2}$ and $B_{2}$ have full rank, the matrix $B_{2} B_{2}^{\top} D_{2}^{\top} D_{2}$ is nonsingular. This implies that $\operatorname{rank}\left(E+B K_{E} D\right)=n$.

Furthermore, it will constructed the conditions such that $\left(E+B K_{E} D\right)^{-1} \in \mathbb{R}_{+}^{n \times n}$ and $\left(A+B K_{A} D\right) \in$ $\mathbb{R}_{+}^{n \times n}$. Consider that

$$
\begin{aligned}
\left(E+B K_{E} D\right)^{-1} & =P\left[\begin{array}{cc}
M_{S} & B_{1} B_{2}^{\top} D_{2}^{\top} D_{2} \\
O & B_{2} B_{2}^{\top} D_{2}^{\top} D_{2}
\end{array}\right]^{-1} Q \\
& =P L Q \\
& =P G H Q,
\end{aligned}
$$

where

$$
\begin{aligned}
L & =\left[\begin{array}{cc}
M_{S} & B_{1} B_{2}^{\top} D_{2}^{\top} D_{2} \\
O & B_{2} B_{2}^{\top} D_{2}^{\top} D_{2}
\end{array}\right]^{-1} \\
& =\left[\begin{array}{cc}
M_{S}^{-1}-M_{S}^{-1} B_{1} B_{2}^{\top} D_{2}^{\top} D_{2}\left(B_{2} B_{2}^{\top} D_{2}^{\top} D_{2}\right)^{-1} \\
O & \left(B_{2} B_{2}^{\top} D_{2}^{\top} D_{2}\right)^{-1}
\end{array}\right], \\
G & =\left[\begin{array}{cc}
M_{S}^{-1} & O \\
O & O
\end{array}\right], \\
H & =\left[\begin{array}{cc}
I_{S}-B_{1} B_{2}^{\top} D_{2}^{\top} D_{2}\left(B_{2} B_{2}^{\top} D_{2}^{\top} D_{2}\right)^{-1} \\
O & \left(B_{2} B_{2}^{\top} D_{2}^{\top} D_{2}\right)^{-1}
\end{array}\right] .
\end{aligned}
$$

Based on Definition 2, it is obvious that $M_{S}^{-1} \in$ $\mathbb{R}_{+}^{s \times s}$ and one can see that if $\left(B_{2} B_{2}^{\top} D_{2}^{\top} D_{2}\right)^{-1} \in$ $\mathbb{R}_{+}^{n \times n}$ and $\left(-B_{1} B_{2}^{\top} D_{2}^{\top} D_{2}\right) \in \mathbb{R}_{+}^{s \times(n-s)}$, then $(E+$ 
$\left.B K_{E} D\right)^{-1} \in \mathbb{R}_{+}^{n \times n}$. Moreover

$$
\begin{aligned}
A+B K_{A} D= & Q^{-1}\left[\begin{array}{ll}
A_{1} & A_{2} \\
A_{3} & A_{4}
\end{array}\right] P^{-1} \\
& +Q^{-1} Q B K_{A} D P P^{-1} \\
= & Q^{-1}\left(\left[\begin{array}{ll}
A_{1} & A_{2} \\
A_{3} & A_{4}
\end{array}\right]\right. \\
& \left.+\left[\begin{array}{l}
B_{1} \\
B_{2}
\end{array}\right] K_{A}\left[O D_{2}\right]\right) P^{-1} \\
= & Q^{-1}\left(\left[\begin{array}{ll}
A_{1} & A_{2} \\
A_{3} & A_{4}
\end{array}\right]\right. \\
& \left.+\left[\begin{array}{ll}
O & B_{1} K_{A} D_{2} \\
O & B_{2} K_{A} D_{2}
\end{array}\right]\right) P^{-1} .
\end{aligned}
$$

One can see that the matrix $K_{A} \in \mathbb{R}^{m \times p}$ can be choose such that

$$
\left(A_{2}+B_{1} K_{A} D_{2}\right) \in \mathbb{R}_{+}^{s \times(n-s)}
$$

and

$$
\left(A_{4}+B_{2} K_{A} D_{2}\right) \in \mathbb{R}_{+}^{(n-s) \times(n-s)} .
$$

Thus we have been proved the following theorem that constitutes a sufficient condition for the existence a fractional derivative output feedback (7).

Theorem 2. Consider the system (1) with $C \in$ $\mathbb{R}_{+}^{n \times q}$ and $\omega \in \mathbb{R}_{+}^{q}$. If there exists the matrices $Q, P \in \mathbb{R}^{n \times n}$ such that $A_{1} \in \mathbb{R}_{+}^{s \times s}, A_{3} \in$ $\mathbb{R}_{+}^{(n-s) \times s},\left(B_{2} B_{2}^{\top} D_{2}^{\top} D_{2}\right)$ is a monomial matrix and $\left(-B_{1} B_{2}^{\top} D_{2}^{\top} D_{2}\right) \in \mathbb{R}_{+}^{s \times(n-s)}$, where $D_{2}$ is a full rank matrix, then there exists the fractional derivative output feedback (7) with $K_{E}, K_{A} \in \mathbb{R}^{m \times p}$ such that $\operatorname{rank}\left(E+B K_{E} D\right)=n$ and the closed loop system (8) is positive.

Compare this with the results in [Muhafzan and $\mathrm{Zu}-$ lakmal, 2017].

The following example illustrates the Theorem 2 . Consider the system (1) with

$$
\begin{aligned}
& E=\left[\begin{array}{lllll}
2 & 0 & 0 & 0 & 0 \\
0 & 2 & 0 & 0 & 0 \\
0 & 0 & 0 & 0 & 0 \\
0 & 0 & 1 & 0 & 0 \\
0 & 0 & 0 & 0 & 0
\end{array}\right], A=\left[\begin{array}{lllll}
1 & 3 & 2 & 1 & 1 \\
3 & 1 & 2 & 1 & 1 \\
1 & 1 & 0 & 4 & 1 \\
1 & 1 & 1 & 1 & 1 \\
1 & 1 & 3 & 0 & 1
\end{array}\right], \\
& B=\left[\begin{array}{ccc}
-0.5 & 0 \\
0 & 0 \\
0 & 1 \\
0 & 0 \\
2 & 0
\end{array}\right], C=\left[\begin{array}{l}
2 \\
0 \\
1 \\
0 \\
1
\end{array}\right] \text { and } D=\left[\begin{array}{lllll}
0 & 0 & 0 & 0 & 1 \\
0 & 0 & 0 & 1 & 0
\end{array}\right] .
\end{aligned}
$$

It is clear that $r=3$ and there exists $\lambda$ such that $\operatorname{det}(\lambda E-A)$. Using the matrices

$$
Q=\left[\begin{array}{lllll}
1 & 0 & 0 & 0 & 0 \\
0 & 1 & 0 & 0 & 0 \\
0 & 0 & 0 & 3 & 0 \\
0 & 0 & 0 & 0 & 1 \\
0 & 0 & 1 & 0 & 0
\end{array}\right] \text { and } P=\left[\begin{array}{lllll}
2 & 0 & 0 & 0 & 0 \\
0 & 1 & 0 & 0 & 0 \\
0 & 0 & 1 & 0 & 0 \\
0 & 0 & 0 & 1 & 0 \\
0 & 0 & 1 & 0 & 1
\end{array}\right]
$$

one can finds that the matrix $E$ is positive equivalent to the matrix

$$
M_{3}=\left[\begin{array}{lll}
4 & 0 & 0 \\
0 & 2 & 0 \\
0 & 0 & 3
\end{array}\right] \text {. }
$$

Furthermore we have

$$
Q B=\left[\begin{array}{cc}
1 & -1 \\
0 & 1 \\
0 & 0 \\
2 & 0 \\
0 & 1
\end{array}\right], D P=\left[\begin{array}{lllll}
0 & 0 & 0 & 0 & 1 \\
0 & 0 & 0 & 1 & 0
\end{array}\right]
$$

and

$$
Q A P=\left[\begin{array}{lllll}
2 & 3 & 2 & 1 & 1 \\
6 & 1 & 2 & 1 & 1 \\
6 & 3 & 3 & 3 & 3 \\
6 & 3 & 3 & 0 & 1 \\
6 & 3 & 0 & 4 & 1
\end{array}\right]
$$

Moreover, it is found

$$
\begin{aligned}
& B_{2} B_{2}^{\top} D_{2}^{\top} D_{2}= {\left[\begin{array}{cc}
0.25 & 0 \\
0 & 1
\end{array}\right], } \\
& B_{1} B_{2}^{\top} D_{2}^{\top} D_{2}=\left[\begin{array}{cc}
-0.5 & 0 \\
0 & 0 \\
0 & 0
\end{array}\right], \\
& A_{2}+B_{1} B_{2}^{\top} D_{2}^{\top} D_{2}=\left[\begin{array}{ll}
3 & 0 \\
1 & 1 \\
3 & 3
\end{array}\right], \\
& A_{4}+B_{2} B_{2}^{\top} D_{2}^{\top} D_{2}=\left[\begin{array}{ll}
4 & 1 \\
4 & 2
\end{array}\right] .
\end{aligned}
$$

Therefore, using

$$
K_{E}=K_{A}=\left[\begin{array}{ll}
0 & 1 \\
2 & 0
\end{array}\right],
$$

one find

$$
\left(E+B K_{E} D\right)^{-1}=\left[\begin{array}{ccccc}
0.5 & 0 & 0 & 0 & 0.125 \\
0 & 0.5 & 0 & 0 & 0 \\
0 & 0 & 0 & 1 & 0 \\
0 & 0 & 0 & 0 & 0.5 \\
0 & 0 & 0.5 & 0 & 0
\end{array}\right] \in \mathbb{R}_{+}^{5 \times 5}
$$


This shows that $\operatorname{rank}\left(E+B K_{E} D\right)^{-1}=n$ and the closed loop system (8) is positive.

\section{Conclusion}

A sufficient condition that ensure the existence of the gain matrices $K_{E}, K_{A} \in \mathbb{R}^{m \times p}$ and the closed loop system (8) is positive has been established. This result constitutes a procedure to normalize positively the fractional order continuous disturbance singular system.

\section{Acknowledgements}

The work was supported by Universitas Andalas under Grant KRP1GB-PDU-Unand-2018 No. 04/UN.16.17/PP.RGB/LPPM/2018.

\section{References}

Chikriy, A. A. and Matichin, I. I. (2008) Presentation of solutions of linear systems with fractional derivatives in sense of Riemann-Liouville, Caputo and Miller Ross. Journal of Automation and Information Sciences, 40(6), pp. 1-11.

Duan, G. R. (2010) Analysis and Design of Descriptor Linear Systems, Springer. London.

Duan, G. R. and Wu, A. G. (2005) Impulse elimination via state feedback in descriptor linear systems, Dynamics of Continuous, Discrete and Impulsive Systems A, 3, pp. 722-729.

Goncharova, E., Samsonyuk, O. and Staritsyn, M. (2017) Dynamical systems with states of bounded pvariation: A new trend in impulsive control, Cybernetics and Physics, 6(4), pp. 208-214.
Kaczorek, T. (2014) Minimum energy control of fractional descriptor positive discrete-time linear systems with bounded inputs, In Proceedings of the 19th World Congress The International Federation of Automatic Control. Cape Town, South Africa, 24-29 August, pp. 2909-2914.

Kaczorek, T. and Borawski, K. (2016) Fractional descriptor continuous-time linear systems described by Caputo-Fabrizio derivative Int. J. Appl. Math. Comput. Sci. 26(3), pp. 533-541.

Kaczorek, T. (2008) Fractional positive continuoustime linear systems and their reachability Int. J. Appl. Math. Comput. Sci. 18(2), pp. 223-228.

Kilbas, A. A., Srivastava, H. M. and Trujillo, J. J. (2006) Theory and Applications of Fractional Differential Equations. Elsevier. Amsterdam.

Metia, S., Oduro, S. D., Duc, H. N. and Ha, Q. (2016) Inverse air-pollutant emission and prediction using extended fractional Kalman filtering IEEE Journal of Selected Topics in Applied Earth Observations and Remote Sensing. 9(5), pp. 2051-2063.

Matviychuk, O. G. (2018) State estimation for bilinear impulsive control systems under uncertainties. $C y$ bernetics and Physics, 7(1), pp. 35-41.

Muhafzan and Stephane, I. (2013) On Stabilization of Positive Linear Systems. Applied Mathematical Sciences, 7(37), pp. 1819-1824.

Muhafzan and Zulakmal (2017) Impulse elimination for positive singular systems using derivative output feedback, In Journal of Physics: Conference Series, Pahang, Malaysia, 8-10 August. 890(012029). 